\title{
MÉTODOS DE AVALIAÇÃO DA FERRUGEM DO ÁLAMO E EFICIÊNCIA DE FUNGICIDAS NO SEU CONTROLE ${ }^{1}$
}

\author{
Louise Larissa May De $\mathrm{Mio}^{2}$ e Lucimeris Ruaro²
}

\begin{abstract}
RESUMO - A ferrugem do álamo (Melampsora medusae Thuem.) causa sérios prejuízos no viveiro, e seu controle é fundamental para a obtenção de muda de boa qualidade. Este trabalho teve como objetivos: i) testar a eficiência de fungicidas de contato (mancozebe, cartap e oxicloreto de cobre) e sistêmicos (triadimenol, tebuconazole e difenoconazole) no controle da ferrugem; ii) comparar métodos de avaliação para discriminar a eficiência entre os tratamentos; iii) relacionar desfolha com dados de doença; e iv) verificar a influência do controle da ferrugem nos parâmetros de crescimento da árvore em viveiro. O experimento foi montado em São Mateus do Sul, PR, delineado inteiramente ao acaso com 10 tratamentos (testemunha, triadimenol, mancozebe, tebuconazole, difenoconazole, cartap, oxicloreto de cobre, triadimenol-mancozebe, tebuconazole-mancozebe e triadimenol aplicados com o dobro do intervalo dos anteriores) e 11 repetições. Com parcelas experimentais de $10 \mathrm{~m}$ de largura com quatro linhas de plantio (espaçamento entre linhas de 2,5 e entre plantas de $0,50 \mathrm{~m}$ ), totalizando 110 parcelas com o clone Latorre. Durante dois ciclos consecutivos foram avaliados: o número de pústulas em meia folha, a \% visual de doença, a severidade por parcela, a \% visual de desfolha, o diâmetro à altura do peito e a altura de plantas ao final do experimento. Os produtos sistêmicos (triadimenol, tebuconazole e difenoconazole) aplicados puros ou intercalados com mancozebe foram eficientes no controle da ferrugem, reduzindo o número de pústulas sobre a folha e a desfolha, o que resultou em ganho significativo no volume final das plantas. O mancozebe aplicado isoladamente também reduziu a epidemia e aumentou o volume da árvore em $42 \%$. O produto cúprico proporcionou aumento de volume em $27 \%$. Os métodos de avaliação utilizados diferenciaram dos tratamentos e houve correlação da doença com os danos na cultura.
\end{abstract}

Palavras-chave: Populus spp., Melampsora medusae e fungicidas.

\section{EVALUATION METHOD FOR POPLAR RUST AND ITS FUNGICIDE CONTROL EFFICIENCY}

\begin{abstract}
Poplar rust (Melampsora medusae Thuem.) causes serious damage in the plant nursery and its control is essential to obtain good quality seedlings. This work aimed at: $i$ ) testing the efficiency of contact fungicides (mancozebe, cartap and copper oxichloride) and systemic fungicides (triadimenol, tebuconazole and difenoconazole) for poplar rust control; ii) comparing evaluation methods to discriminate the efficiency among the treatments; iii) relating defoliation and disease data and iv) verify the influence of rust control under growth tree parameters. The experiment was conducted in São Mateus do Sul, PR and arranged in a completely randomized design with 10 treatments (check, triadimenol, mancozebe, tebuconazole, difenoconazole, cartap, copper oxichloride, triadimenol-mancozebe, tebuconazole-mancozebe and triadimenol applied at double the interval of the previous ones) and 11 repetitions. The experimental plots were $10 \mathrm{~m}$ wide with 4 planting rows (space between the lines was 2.5 and between the plants, $0.50 \mathrm{~m}$ ), totaling 110 plots with the Latorre clone. The following aspects were evaluated during two consecutive cycles: number of pustules per half leaf; visual percentage of the disease; severity per plot; visual percentage of defoliation, diameter at breast height and plant height at the end of the experiment. The systemic products (triadimenol, tebuconazole and difenoconazole) applied alone or interpolated with mancozebe were efficient in controlling the disease,
\end{abstract}

\footnotetext{
${ }^{1}$ Recebido em 24.03.2007 e aceito para publicação em 22.08.2008.

${ }^{2}$ Departamento de Fitotecnia e Fitossanitarismo da Universidade Federal do Paraná (UFPR). E-mail:<maydemio@ufpr.br>
} 
reducing both the number of leaf pustules and defoliation, resulting in a significant gain in the final plant volume. Mancozebe applied alone also reduced epidemics and increased tree volume in $42 \%$. The cupric product made the volume increase in $27 \%$. The evaluation methods used showed differences among the treatments and there was a correlation between the disease and the damage caused to the culture.

Keywords: Populus spp., Melampsora medusae and fungicides.

\section{INTRODUÇÃO}

O álamo (Populus spp.) é uma espécie florestal que agrega alto valor econômico, e sua madeira clara e resistente é utilizada principalmente para fabricação de palito de fósforo. No Paraná, os plantios comerciais estão localizados no vale do Iguaçu e contam com uma área de aproximadamente 3.000 ha.

Os plantios no Vale do Rio Iguaçu normalmente são feitos com mudas de dois anos de viveiro. O problema fitossanitário mais grave no viveiro é a ferrugem, ocasionada pelo fungo Melampsora medusae Thuem. Os sintomas apresentados pelas plantas atacadas são pústulas de coloração amarela a alaranjada, que se distribuem pelas superfícies inferior e superior da folha. Em alguns casos, as pústulas se unem e formam, posteriormente, regiões necrosadas, levando à queda prematura das folhas. Os sintomas aparecem, geralmente, no final da primavera, progredindo até o final do verão, aonde se inicia o processo de desfolha natural. Essa desfolha contribui para o depauperamento da árvore e impede a planta de armazenar energia para o inverno. O resultado disso é notado no ano seguinte, com acentuada perda de crescimento, predispondo a planta ao ataque de outros insetos e doenças (PAILLASSA, 1996; YIN et al., 2004). A utilização de clones de álamo resistentes à ferrugem tem sido recomendada (NEWCOMBE et al., 1996). Mas a maioria dos clones comercialmente utilizados (maioria híbridos de $P$. deltóides Bartr. ex. Marsh) são altamente suscetíveis às doenças (SPIERS, 1975; MCCRACKEN et al., 1984; PRAKASH e HEATHER, 1986; NEWCOMBE e CHASTAGNER, 1993; MAY De MIO, 1997).

A doença, quando atinge níveis epidêmicos, pode causar sérios danos à cultura, interferindo na produção e qualidade da madeira (WIDIN e SCHIPPER JÚNIOR, 1981; STEENACKERS et al., 1995). No Vale do Rio Iguaçu, $\mathrm{PR}$, região do plantio de álamo no Brasil, os clones têm sido infectados com ferrugem, e a epidemia é severa em anos com temperaturas mais amenas no verão.

Existe demanda por parte dos produtores em pesquisa, principalmente no controle da doença no viveiro, para possibilitar a produção de muda de qualidade para iniciar o plantio definitivo. Os produtores têm observado que as epidemias, crescentes ao longo dos anos, têm prejudicado o desenvolvimento das mudas. Além disso, é comum observar mudanças no comportamento dos clones em relação à suscetibilidade à ferrugem. $\mathrm{O}$ controle no viveiro em anos de epidemia severa pode garantir a manutenção da folha por mais tempo e, assim, a planta guardará mais energia para passar o inverno. Nos plantios comerciais dos países produtores em todo o mundo não se controla a doença com fungicidas, mas sim com manejo e escolha de clones menos suscetíveis, entretanto isso depende de constante pesquisa em melhoramento genético. No Brasil, este trabalho ainda não vem sendo realizado por órgãos oficiais de pesquisa e o comportamento da epidemia em diferentes clones, bem como o controle químico, já se mostra viável para uso em viveiro (MAY De MIO et al., 2002).

A limitação está em buscar alternativas de controle e para manejar a doença de forma ecológica, eficiente e econômica. Essa doença, quando não controlada, reduz drasticamente a produção de madeira com perdas estimadas ao redor de $60 \%$ no viveiro (MAY De MIO et al., 2002).

Este trabalho teve como objetivos: i) testar a eficiência de fungicidas de contato (mancozebe, cartap e oxicloreto de cobre) e sistêmicos (triadimenol, tebuconazole e difenoconazole) para o controle da ferrugem; ii) comparar métodos de avaliação para discriminar a eficiência entre os tratamentos; iii) relacionar desfolha com dados de doença; e iv) verificar influência do controle da ferrugem nos parâmetros de crescimento da árvore em viveiro.

\section{MATERIAL E MÉTODOS}

\subsection{Experimento de campo}

O experimento foi realizado na Fazenda São Joaquim, da empresa Andrade Latorre, localizada no Município de São Mateus do Sul, PR, na região do Vale do Rio 
Iguaçu, em área de viveiro do clone Latorre, durante dois anos consecutivos.

O experimento foi delineado inteiramente ao acaso com 10 tratamentos, utilizando-se os seguintes ingredientes ativos ( $\mathrm{g}, \mathrm{kg}$ ou $\mathrm{ml} 100 \mathrm{~L}^{-1}$ de água): triadimenol $(250 \mathrm{ml})$; mancozebe $(1,5 \mathrm{~kg})$; tebuconazole ( $250 \mathrm{ml})$; difenoconazole $(21 \mathrm{ml})$; cartap $(160 \mathrm{~g})$; oxicloreto de cobre $(600 \mathrm{~g})$; triadimenol-mancozebe $(250 \mathrm{ml}$ e 1,5 $\mathrm{kg}$, respectivamente); tebuconazole-mancozebe (250 $\mathrm{ml} \mathrm{e} 1,5 \mathrm{~kg}$, respectivamente); triadimenol aplicado com o dobro do intervalo dos anteriores $(250 \mathrm{ml})$; e tratamento testemunha.

Foram utilizadas 11 repetições e parcelas experimentais de $10 \mathrm{~m}$ de largura com quatro linhas de plantio (espaçamento entre linhas de 2,5 m e entre plantas de $0,50 \mathrm{~m}$ ), totalizando 110 parcelas em uma área de 1,1 ha com o clone "Latorre", altamente suscetível à ferrugem (MAY De MIO e AMORIM, 2001).

Nos tratamentos com triadimenol-mancozebe e naqueles com tebuconazole-mancozebe, os fungicidas foram intercalados a cada aplicação. Pulverizaram-se os produtos de 15 a 20 dias em função clima, sendo feitas quatro pulverizações no primeiro ano (de novembro a fevereiro) e sete no segundo (de novembro a abril), realizadas com pulverizador tratorizado tipo canhão, numa vazão de 200 L.ha' ${ }^{-1}$.

\subsection{Metodologias de avaliação da ferrugem}

As avaliações foram iniciadas no mês de dezembro com intervalo mensal, estendendo-se até março, medindose como parâmetros de avaliações da doença as seguintes variáveis: 1) número de pústulas em meia folha na quinta árvore de cada parcela, nas folhas posicionadas a aproximadamente $1 \mathrm{~m}$ do solo, aos sete dias após a segunda pulverização - essa avaliação foi realizada apenas no primeiro ano; 2) porcentagem visual de doença na parcela, estimada mensalmente de dezembro a fevereiro no primeiro ano e apenas em março no segundo ano; e 3) severidade da doença por escala de nota, realizada apenas uma vez em março do primeiro ano de avaliação. As notas variaram de 0 a $5(0=$ ausência de ferrugem; $1=$ presença leve no terço inferior da planta, porém sem necroses; 2 = ataque moderado no terço inferior sem necroses, porém com o terço superior intacto; 3 = ferrugem ao longo da planta com necroses no terço inferior; 4 = ataque severo na planta, porém com necroses e folhas mortas com índices menores que 50\%; e 5 $=$ ataque severo por toda a planta com mais de $50 \%$ de folhas com necroses e mortas), avaliados nas quatro plantas centrais de cada parcela (STEENACKERS et al., 1994).

Como parâmetros diretamente relacionados ao efeito da epidemia na fisiologia e crescimento da planta, avaliaram-se os seguintes fatores: 1) porcentagem visual de desfolha em cada parcela em maio do segundo ano, um mês após a última pulverização; e 2) diâmetro à altura do peito e altura de duas plantas por parcela na ocasião do transplante destas para o local definitivo, ao final do segundo ano de viveiro (no mês de agosto).

Nas avaliações que foram realizadas ao longo do tempo em diferentes datas, os dados foram integralizados no tempo e transformados em área sobre a curva do progresso da variável relacionada (ASCPD), seguindo-se metodologia da integralização trapezoidal (BERGER, 1988).

\subsection{Análise dos dados}

$\mathrm{Na}$ análise estatística foram consideradas as variáveis: \% da desfolha, número de pústulas em metade de uma folha, severidade da doença (escala de notas), área sobre a curva da doença (ASCPD) da \% visual de doença na parcela. O modelo ajustado considerou os fatores em delineamento inteiramente ao acaso, com 11 repetições e 10 tratamentos.

O pressuposto de normalidade foi verificado nas variáveis de interesse, e procedeu-se à transformação Box-Cox, nos casos que se fizeram necessárias.

Os dados de desfolha foram correlacionados com dados de doença e desenvolvimento da planta por regressões lineares, utilizando-se o programa Excel 6.0.

\section{RESULTADOS E DISCUSSÃO}

A infecção da ferrugem no primeiro ano começou cedo, aos sete dias após a segunda aplicação dos fungicidas, em meados de dezembro. O número de pústulas em meia folha (amostragem) já estava elevado com valores entre 6 e 100 pústulas em cada amostra. Nessa etapa, verificou-se muita variabilidade entre as parcelas, sendo essa metodologia desconsiderada nas próximas avaliações. Outro fato importante foi que as folhas escolhidas para avaliação estavam posicionadas a aproximadamente $1 \mathrm{~m}$ do solo, e elas caíam facilmente a partir do mês de janeiro, o que dificultava manter uma avaliação não destrutiva na mesma folha ao longo do tempo.

R. Árvore, Viçosa-MG, v.32, n.5, p.837-844, 2008 
No segundo ano, a epidemia iniciou-se em dezembro, aumentando em severidade até o mês de fevereiro, que culminou com as condições favoráveis para o crescimento do patógeno. Ao final de março, a doença estabilizou-se e iniciou a queda das folhas, quando o patógeno passou por uma fase de sobrevivência até o próximo ciclo.

Os tratamentos com uso de triadimenol, de mancozebe, de tebuconazole e de difenoconazole de forma geral foram eficientes, retardando a entrada da ferrugem para o início do mês de janeiro.

A variabilidade observada foi elevada, com grande quantidade de observações de valor zero (94 em 110 observações). Os tratamentos em que se verificaram valores diferentes de zero foram: testemunha; cartap; oxicloreto de cobre e triadimenol, sendo, em média, notados 13,$4 ; 34,5 ; 26,6$; e 31,8 pústulas em meia folha, respectivamente. O menor valor observado foi 1 e o maior, 100 pústulas em meia folha, sendo a amplitude observada de 99 pústulas. Os demais tratamentos não apresentavam sintomas na folha avaliada. $\mathrm{O}$ valor mais alto do tratamento com triadimenol pode ter sido devido à fitotoxicidade provocada por uma aplicação de fentin acetato, que tinha sido aplicado na primeira pulverização; esse tratamento foi, então, substituído por triadimenol, com intervalo de 30 dias a partir da segunda pulverização. Tal efeito pode ter favorecido a entrada da epidemia.

A porcentagem visual da doença ao final do experimento estava elevada (32\% no primeiro ano e $44 \%$ no segundo), o que contribuiu, positivamente, para a diferenciação quanto à eficiência dos produtos aplicados. Nos tratamentos com triadimenol aplicado sozinho ou intercalado com mancozebe, a doença não evoluiu. Nos demais tratamentos, também a doença foi controlada em porcentagens, que variaram de 68 a $91 \%$ no primeiro ano e 49 e $96 \%$ no segundo em relação à testemunha, sendo nesse caso o melhor tratamento o tebuconazole e o pior, o cartap.

$\mathrm{Na}$ análise estatística, a variável ASCPD da porcentagem visual da doença mostrou efeito significativo do fator tratamento. As médias dos tratamentos podem ser vistas na Tabela 1 .

A análise de variância indicou efeito altamente significativo dos tratamentos na porcentagem de desfolha (Tabela 1). A desfolha provocada pela ferrugem nessa cultura tem sido destacada em alguns trabalhos das literaturas internacional e nacional (WIDIN e SCHIPPER JÚNIOR, 1981; HAMELIN et al., 1993; MAY De MIO et al., 2002). Em casos extremos, como relataram alguns trabalhos, desfolhas repetidas, principalmente em árvores jovens em desenvolvimento, podem reduzir reservas nas raízes e predispor as árvores a outros patógenos (SCHIPPER e DAWSON, 1974).

Os valores da severidade da doença nos tratamentos com fungicidas foram menores que na testemunha (Tabela 2). A eficiência desse método também não foi ideal, porque os níveis mais elevados de nota (3|4) não apresentaram padronização significativa no nível de $5 \%$ de probabilidade, não sendo indicado como bom parâmetro para avaliação.

A concentração de notas maiores significa maior severidade de danos na testemunha, todos os tratamentos à base de triazóis (triadimenol, tebuconazole, triadimenolmancozebe e tebuconazole-mancozebe) apresentaram as menores notas (menos doença). Em concordância com esses dados está o trabalho de Wu Hong Yuan et al. (1993), que obtiveram $85 \%$ de controle da ferrugem do álamo com triazóis. Outros autores como Giorcelli e Vietto (1995) também comprovaram a eficiência de triazóis usando o ingrediente ativo triadimenol.

Tabela 1 - Área sob a curva de progresso da doença (ASCPD), em porcentagem visual da doença e porcentagem de desfolha em diferentes tratamentos, para o controle da ferrugem do álamo, São Mateus do Sul, PR

Table 1 -Area under disease progress curve (AUDPC) in visual percentage of the disease and defoliation percentage in different treatments to control poplar rust, São Mateus do Sul, PR

\begin{tabular}{lcc}
\hline $\begin{array}{l}\text { Tratamentos } \\
\text { (ingrediente ativo) }\end{array}$ & ASCPD $^{3}$ & ${\text { Desfolha }(\%)^{1}}$ \\
\hline Testemunha & $2677,0 \mathrm{e}$ & $70,91 \mathrm{c}$ \\
Cartap & $1122,0 \mathrm{~d}$ & $55,45 \mathrm{c}$ \\
Oxicloreto de cobre & $737,5 \mathrm{~cd}$ & $32,27 \mathrm{ab}$ \\
Triadimenol & $606,5 \mathrm{c}$ & $32,73 \mathrm{~b}$ \\
difenoconazole & $523,0 \mathrm{c}$ & $28,18 \mathrm{ab}$ \\
mancozebe $_{\text {Triadimenol }}{ }^{1}$ & $470,0 \mathrm{bc}$ & $25,91 \mathrm{ab}$ \\
Triadimenol - mancozebe $^{2}$ & $185,0 \mathrm{ab}$ & $26,82 \mathrm{ab}$ \\
Tebuconazole - mancozebe & $157,5 \mathrm{ab}$ & $21,82 \mathrm{a}$ \\
Tebuconazole & $112,5 \mathrm{ab}$ & $27,73 \mathrm{ab}$ \\
\hline
\end{tabular}

${ }^{1}$ Aplicação com intervalo de 30 dias.

Aplicados intercalados a cada 15 dias

${ }^{3}$ Letras indicam diferenças pelo teste de Tukey a 5\% de probabilidade. 
Tabela 2 - Número de observações usando escala de nota para avaliação da severidade da ferrugem do álamo com diferentes tratamentos para seu controle

Table 2-Number of observations using note scale to assess severity of poplar rust with different control treatments

\begin{tabular}{llllll}
\hline Tratamento & \multicolumn{5}{c}{ Escala de Notas $^{1}$} \\
\cline { 2 - 6 } & 0 & 1 & 2 & 3 & 4 \\
\hline Testemunha & 0 & 0 & 2 & 4 & 5 \\
Triadimenol & 7 & 4 & 0 & 0 & 0 \\
Mancozebe & 3 & 5 & 3 & 0 & 0 \\
Tebuconazole & 9 & 2 & 0 & 0 & 0 \\
Difenoconazole & 2 & 6 & 3 & 0 & 0 \\
Cartap & 0 & 5 & 2 & 4 & 0 \\
Oxicloreto de cobre & 0 & 7 & 4 & 0 & 0 \\
Triadim-mancozebe & 8 & 3 & 0 & 0 & 0 \\
Tebuc-mancozebe & 6 & 4 & 1 & 0 & 0 \\
Triadimenol $^{2}$ & 0 & 3 & 6 & 2 & 0 \\
\hline
\end{tabular}

${ }^{1}$ Steenackers et al., 1995, notas aumentam de severidade de 0 a 4. ${ }^{2}$ Aplicado a cada 30 dias.

A maioria dos produtos testados foi eficiente no controle da $M$. medusae, o que foi evidenciado, principalmente, com o volume de madeira produzido pelas plantas nos diferentes tratamentos. Uma planta que perde as folhas no inverno armazena energia ao final do seu ciclo (maio/junho), para que no próximo ano possa brotar abundantemente e continuar a crescer. Se esse processo é adiantado, menor quantidade de reserva é armazenada e, consequientemente, o crescimento da planta no próximo ano é menor, conforme afirmaram Widin e Schipper Júnior (1981), os quais verificaram que a desfolha causada por Melampsora em híbridos suscetíveis reduziu $30 \%$ do peso de matéria seca e $42 \%$ do volume da madeira. Outros autores como Tabor et al. (2000) observaram redução de $60 \%$ na produção de madeira, e Dowkiw et al. (2002) relataram perdas anuais de $50 \%$ do crescimento de plantas atacadas pela ferrugem.

Quanto à variável AACPD do DAP, houve efeito significativo do fator tratamento. No entanto, aqui estão apresentados os dados médios dos parâmetros DAP, altura e volume de madeira em cada parcela, por tratamento, ao final do experimento (Tabela 3 ).

Os tratamentos que proporcionaram maior crescimento da planta foram, em ordem decrescente: triadimenol intercalado com mancozebe, mancozebe, triadimenol, tebuconazole e tebuconazole intercalado com mancozebe (Tabela 3). No entanto, todos os tratamentos proporcionaram incrementos na produção de 26 a $42 \%$. A ferrugem pode ter pouco efeito no crescimento de plantas de álamo na primeira fase de crescimento. Porém, no ano seguinte à epidemia, o diâmetro e a altura da planta são reduzidos (WILCOX e FARMER, 1967). A infecção por Melampsora medusae provoca desfolha prematura e redução no crescimento (TOOLE, 1967; WIDIN e SCHIPPER JÚNIOR, 1976; COOPER e FILER JÚNIOR, 1977; STEENACKERS et al., 1995). A atividade fotossintética é reduzida, resultando em diminuição do crescimento em diâmetro e altura e no volume de madeira produzido (PAILLASSA, 1996). Também são constatadas alterações na qualidade da madeira e diminuição das substâncias de reservas armazenadas. Todo esse prejuízo é tanto maior quanto mais precoce for o ataque no ciclo de cultivo (STEENACHERS et al., 1995). No Brasil, May de Mio et al. (2002) observaram redução em $30 \%$ no volume de madeira (considerando a muda produzida em dois anos) em clones suscetíveis, no viveiro, na ausência de controle químico com fungicida triazol.

A estimativa visual da doença não foi correlacionada com porcentagem de desfolha ao final do segundo ano com índice de correlação alto $\left(\mathrm{R}^{2}=0,91\right)$, quando foram considerados os dados médios entre as 11 repetições (Figura 1a).

Nesse caso, fica evidente a separação entre os dados dos tratamentos que receberam fungicidas e o tratamento-testemunha, com relação direta entre alta quantidade de doença e alta desfolha. Conforme Hamelin et al. (1993), a doença é subestimada quando a severidade é a única variável avaliada, porque ao final do ciclo, quando as folhas altamente infectadas já caíram, a severidade nas folhas remanescentes é baixa.

Considerando os dados totais, a regressão linear entre a \% visual de doença no segundo ano (final de março) e os dados de desfolha do próximo mês resultaram $R^{2}=0,53$ (Figura 1b). Esse fato representa que a doença, mesmo sendo controlada, explica 53\% da desfolha antecipada.

Essa doença, como pode ser evidenciado neste trabalho, é limitante da produção de álamos nas condições de cultivo no vale do rio Iguaçu, PR. Apesar de difícil registro de produtos para culturas de baixa expressão econômica, os produtores têm incentivado pesquisa e estado sempre em contato com a Secretaria de Agricultura e com as empresas de fungicidas, no intuito de conseguir registros de novos produtos para a cultura. Atualmente, apenas o fungicida triadimenol está liberado para uso no Paraná (Secretaria Estadual de Agricultura e do Abastecimento do Estado do Paraná-SEAB, março, 2006).

R. Árvore, Viçosa-MG, v.32, n.5, p.837-844, 2008 
Tabela 3 - Diâmetro à altura do peito, altura da árvore, volume total de madeira e aumento relativo de volume de madeira de álamo em viveiro, em diferentes tratamentos para controle da ferrugem

Table 3 -Diameter of breast height, tree height, total poplar wood volume and poplar wood relative increase in the nursery, in different treatments, for rust control

\begin{tabular}{|c|c|c|c|c|}
\hline \multirow[t]{2}{*}{ Tratamentos } & \multicolumn{4}{|c|}{ Avaliações ${ }^{1}$} \\
\hline & $\mathrm{DAP}(\mathrm{cm})$ & $\operatorname{ALTURA}(\mathrm{cm})$ & Volume $\left(\mathrm{cm}^{3}\right)$ & Aumento do volume (\%) \\
\hline Testemunha & 4,90 & 676,00 & 5853,70 & 19,3 \\
\hline Triadimenol & 6,01 & 734,36 & 9563,58 & 37,5 \\
\hline Mancozebe & 6,12 & 763,45 & 10306,70 & 42,5 \\
\hline Tebuconazole & 5,91 & 745,82 & 9406,63 & 37,4 \\
\hline Difenoconazole & 5,77 & 741,73 & 8914,52 & 33,4 \\
\hline Cartap & 5,35 & 691,60 & 7125,94 & 23,5 \\
\hline Oxicloreto de cobre & 5,55 & 733,09 & 8143,96 & 26,9 \\
\hline Triadimenol-mancozebe & 6,33 & 791,60 & 11439,45 & 42,6 \\
\hline Tebuconazole-mancozebe & 5,88 & 747,00 & 9308,90 & 35,9 \\
\hline Triadimenol 30dias & 5,43 & 677,73 & 7211,69 & 27,8 \\
\hline
\end{tabular}

${ }^{1}$ Valor médio de duas plantas por parcela, sendo 11 parcelas avaliadas/tratamento.

${ }^{2}$ Volume das árvores calculado pela multiplicação do cilindro perfeito pelo Ff do clone - LATORRE, $0,4592$.
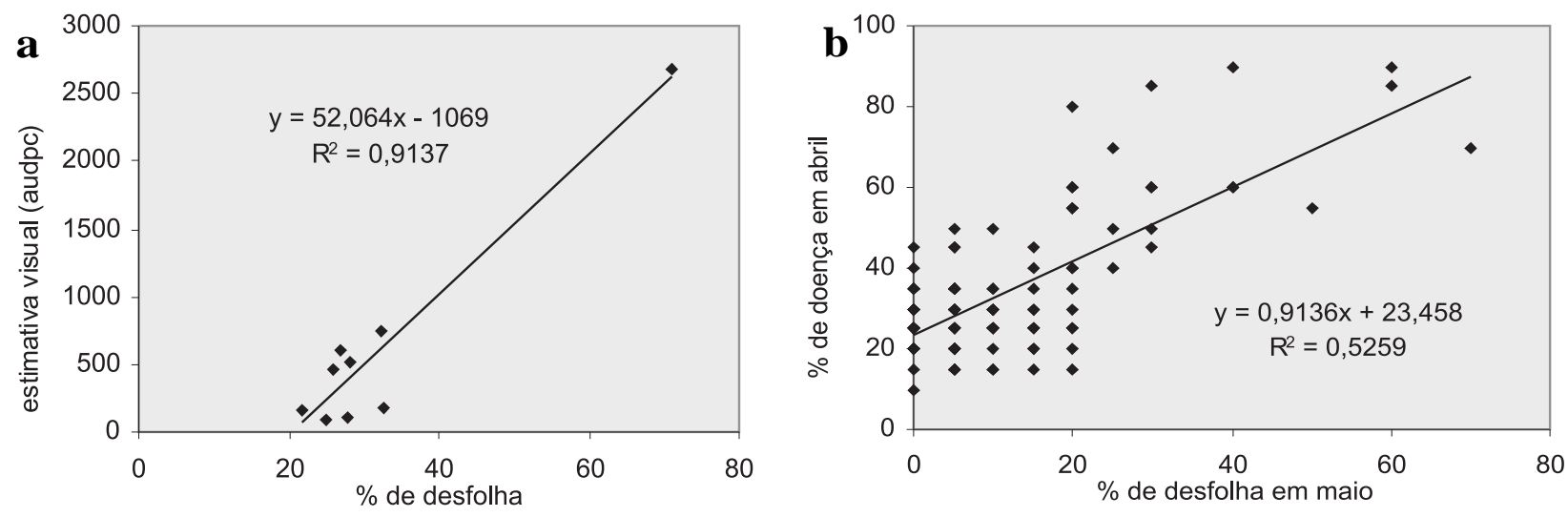

Figura 1 - Relação entre a desfolha em maio e a estimativa visual da ferrugem em AACPD do ano anterior (a) e em abril do mesmo ano (b), em viveiro do clone Latorre, São Mateus do Sul, PR.

Figure 1 -Relationship between defoliation in May and visual estimate of rust in AUDPC a year before (a) and in Abril of the same year (b), in Latorre clone nursery, São Mateus do Sul, PR.

\section{CONCLUSÕES}

Os produtos triazóis (triadimenol, tebuconazole e difenoconazole) aplicados puros ou intercalados com mancozebe foram eficientes no controle da ferrugem, reduzindo o número de pústulas sobre a folha e a porcentagem visual de desfolha, o que resultou em ganho significativo no volume final das plantas.

O mancozebe aplicado isoladamente também reduziu a epidemia e aumentou o volume da árvore em $42 \%$. O produto cúprico, apesar de não ser eficiente como os demais testados, proporcionou aumento de $27 \%$ em volume.
Em relação aos métodos de avaliação estudados, as estimativas visuais da doença e da desfolha diferenciaram os tratamentos.

A estimativa visual da ferrugem em abril e a área abaixo da curva da estimativa visual tiveram correlação com a desfolha avaliada no mês de maio.

\section{REFERÊNCIAS}

BERGAMIN FILHO, A. et al. Avaliação de danos causados por doenças de plantas. Revisão Anual de Patologia de Plantas, v.3, p.133-184, 1995. 
BERGER, R. D. The analysis of the effects of control measures on the development of epidemics. In: KRANZ, J.; ROTEM, J. (Eds.). Experimental techniques in plant disease epidemiology. Heidelberg: SpringerVerlang, 1988. p.137-151.

COOPER, D. T.; FILER JÚNIOR, T. H. Geographic variation in Melampsora rust resistance in eastern cottonwood in the lower Mississippi valley. In: CENTRAL STATES FOREST TREE

IMPROVEMENT CONFERENCE, 1976, St. Paul. Proceedings... St. Paul: USDA, Forest Service, 1977. p.146-151.

DOWKIW, A. et al. Partial resistance to Melampsora larici-populina leaf rust in hybrid poplars: genetic variability in inoculated excised leaf disk bioassay and relationship with complete resistance.

Phytopathology, v.93, n.4, p.421-427, 2003.

GIORCELLI, A.; VIETTO, L. Valutatazione delléfficacia di alcunio fungicidi versu le ruggini del pioppo. Informatore Fitopatologico, v.45, n.6, p.59-62, 1995.

HAMELIN, R. C. et al. Quantification of disease progress and defoliation in poplar leaf rusteastern cottonwood pathosystem.

Phytopathology, v.83, n.2, p.140-144, 1993.

MAY-DE MIO, L. L. Identificação das principais doenças do álamo (Populus spp) e epidemiologia e controle químico da ferrugem (Melampsora medusae Thüm). Curitiba: Universidade Federal do Paraná, 1997. 64 p. (Relatório técnico apresentado à Fundação de Pesquisas em Florestas da Universidade Federal do Paraná).

MAY-DE MIO, L. L.; AMORIM, L. Influência da temperatura e do molhamento foliar nos componentes monocíclicos da ferrugem do álamo. In: CONGRESSO BRASILEIRODE

FITOPATOLOGIA, 43., 2001, Águas de São Pedro. Fitopatologia Brasileira, Suplemento, p.232, 2001.

MAY DE MIO, L. L.; AMORIM, L.; SCHUTA, L. R. Suscetibilidade de clones de álamo à ferrugem no viveiro, eficiência de fungicidas e avaliação de danos. Summa Phytopathologica, v.28, n.1, p.46-51, 2002.
McCRACKEN, F. I.; SCHIPPER, A. L.; WIDIN, K. D. Observation on occurrence of cottonwood leaf rust in central United States. European Journal of Forest Pathology, v.14, N.4/5, p.226-233, 1984.

NEWCOMBE, G.; CHASTAGNER, G. A. A leaf rust epidemic of hybrid poplar along the lower Columbia River caused by Melampsora medusae. Plant Disease, v.77, n.5, p.528-531, 1993.

PAILLASSA, E. Les roilles et le peuplier. Les Cahiers Techniques de l'Objetif 1, Bulletin Trimestriel. Centre de Populiculture du Hainaut, n.2, p.9-18, 1996.

PRAKASH, C. S.; HEATHER, W. A. Effects of changing temperature regimes on resistance to races of Melampsora medusae in cultivar of poplar. Annals Applied Biology, v.108, n.2, p.403-407, 1986 .

SCHIPPER, A. L.; DAWSON, D. H. Poplar leaf rust - A problem in maximum wood fiber production. Plant Disease Reporter, v.58, p.721-723, 1974.

SPIERS, A. G. Species of Melampsora infecting conifers in New Zealand. Plant Disease Reporter, v.59, p.486-488, 1975.

STEENACHERS, M.; STEENACHERS, V.; DELPORT, T. A new physiological race of Melampsora larici-populina in Belgium. In: FAOCIP MEETINGS OF THE WORKING GROUP OF DISEASES, 1994, Turkey. FAO...Turkey: FAO/ CIP, 1994. 6p.

STEENACHERS, J.; STEENACHERS M.; STEENACHERS, V. Maladies des peupliers Consequences sur la croissance et la qualite du bois. Les Cahiers Techniques de l'Objetif, v.1, p.4-20, 1995.

TABOR, G. M. et al. Bulked segregant analysis identifies molecular markers linked to Melampsora medusae resistance in Populus deltoids.

Phytopathology, v.90, n.9, p.1039-1042, 2000.

TOOLE, E. R. Melampsora medusae causes cottonwood rust in lower Mississipi valley. Phytopathology, v.57, p.1361-1362, 1967.

R. Árvore, Viçosa-MG, v.32, n.5, p.837-844, 2008 
WIDIN; K. D.; SCHIPPER JÚNIOR, A. L. Epidemiology and impact of Melampsora medusae leaf rust on hybrid poplars. In: INTENSIVE plantation culture-five years research. St. Paul: Dep. Agric. For. Serv., North Cent. For. Exp. Stn., 1976. p.63-74. (U.S. For. Serv. Gen. Tech. Rep. NC-21).

WIDIN, K. D.; SCHIPPER JÚNIOR, A. L. Effect of Melampsora medusae leaf rust infection on yield of hibrids poplars in the north central United States. European Journal of Forestry Pathology, v.11, n.7, p.438-448, 1981.

WILCOX, J. R.; FARMER, R. E. J. Variation and inheritance of juvenile characters of eastern cottonwood. Silvae Genetic, v.16, p.162-165, 1967.
WAGGONER, P. E.; BERGER, R. D. Defoliation, disease and growth. Phytopathology, v.77, n.33, p.393-398, 1987.

WU-HONG, Y. et al. Studies on the control measures of poplar rust, Melampsora laricipopulina in China. Impact of diseases and insect pests in tropical forest. In: IUFRO SYMPOSIUM, Peechi, 1993.

Proceedings...Peechi: Kerala Forest Research Institute, 1996. p.186-191.

YIN, T. M. et al. Genetic and physical mapping of Melampsora rust resistance genes in Populus and characterization of linkage disequilibrium and flanking genomic sequence. New Phytologist, v.164, n.1, p.95-105, 2004. 